\title{
Discussion of "Observation of Ground Movement with Existing Pile Groups Due to Tunneling in Sand Using Centrifuge Modelling” by Ittichai Boonsiri and Jiro Takemura
}

\author{
Alec M. Marshall $[$ • Andrea Franza
}

Received: 1 March 2016/Accepted: 24 August 2016/Published online: 2 September 2016

(C) The Author(s) 2016. This article is published with open access at Springerlink.com

\begin{abstract}
This discussion aims to highlight the underlying cause of several aspects of the greenfield settlement data presented by Boonsiri and Takemura (Geotech Geol Eng 33(3):621-640, 2015). The discussion considers, for the geotechnical centrifuge tests that were reported, the effects of the boundary conditions imposed at the model tunnel on resulting settlements. Data obtained using the rigid boundary model tunnel in Boonsiri and Takemura (Geotech Geol Eng 33(3):621-640, 2015) are compared against other available data from tests using a fluid-filled flexible membrane model tunnel. It is demonstrated that the boundary conditions used to simulate tunnel ground loss have an important impact on the settlement mechanism; compared to a fluid-filled flexible membrane, a rigid boundary model tunnel results in wider settlement troughs, which do not vary in shape considerably with changes in relative tunnel depth, and can result in higher ratios between the area of the settlement troughs and the tunnel ground loss. The appropriateness of the different tunnel boundary conditions is also discussed.
\end{abstract}

Keywords Tunnel - Centrifuge - Displacements . Greenfield

A. M. Marshall $(\bowtie) \cdot$ A. Franza

University of Nottingham, Nottingham, UK

e-mail: alec.marshall@nottingham.ac.uk
Boonsiri and Takemura (2015) present a valuable dataset obtained from geotechnical centrifuge testing of the effect of tunnelling on piles. This is undoubtedly an important engineering problem and the data provided gives a good indication of the complex interactions that take place between the tunnel, soil, and pile.

This discussion aims to look more closely at the greenfield data presented by the authors and the comparison to published relationships for the prediction of the shape of greenfield settlements in sands. The available data in the literature for the trough width parameter, $K$, in sands are characterised by a high level of scatter that must, in some part, be due to the complexity of the soil response to underground excavation and its dependency on multiple factors. Past studies demonstrated that the settlement curve shape and volume (i.e. the width parameter $K$ and the soil volume loss $V_{l, s}$, respectively) are affected by the cover to diameter ratio, $C / D$, the magnitude of tunnel volume loss, $V_{l, t}$, and the soil relative density, $I_{d}$ (Sugiyama et al. 1999; Marshall et al. 2012; Zhou et al. 2014; Franza and Marshall 2015). This dependency is confirmed by the greenfield centrifuge test data presented by Boonsiri and Takemura (2015) (referred to as $\mathrm{B} \& \mathrm{~T})$. B\&T showed that the obtained greenfield data fits well to the relationship proposed by Moh et al. (1996), which was based on settlement data from a single tunnelling project in Taipei and, therefore, is not able to account for the effect of the range of 


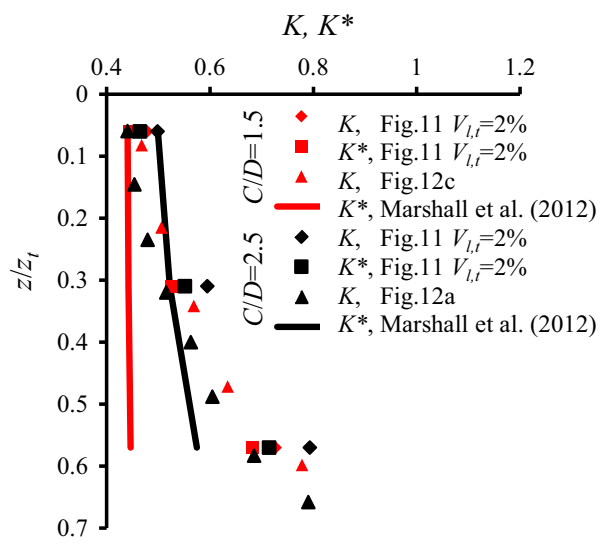

Fig. 1 Variation of settlement width parameters with normalized depth

influencing parameters. The set of equations provided by Marshall et al. (2012) were, like the data presented by $\mathrm{B} \& \mathrm{~T}$, based on data from centrifuge experiments conducted using a dry, fine-grained silica sand. These equations, however, did not provide a good prediction of the dataset presented by B\&T. It is worth investigating the possible reasons that could explain why the results of these two series of tests differ. The data provided in B\&T (Figs. 11 and 12) was digitized and has been reproduced in this discussion in order to compare to other available data. The discussion focuses on the data obtained at a volume loss of $2 \%$ since, among the two values of volume loss considered in the paper, this is the most applicable to realistic conditions (the other volume loss being $14 \%$ which is higher than reasonably expected). All curve fitting was done using standard least-squares regression techniques within Matlab.

Gaussian and modified Gaussian curves were fitted to the data provided in Fig. 11 of B\&T; the resulting values of $K$ and $K^{*}$ (Marshall et al. 2012) are compared with the results displayed in B\&T Fig. 12a $(C / D=2.5)$ and Fig. $12 \mathrm{c}(C / D=1.5)$ in Fig. 1. The obtained values of $K$ and $K^{*}$ match reasonably well to the data provided by B\&T. The estimations of $K^{*}$ based on the relationship suggested by Marshall et al. (2012) is also plotted. Interestingly, there is an acceptable agreement between the centrifuge outcomes and the Marshall et al. (2012) predictions for the tunnel with $C / D=2.5$, whereas the prediction of subsurface values is unsatisfactory for $C / D=1.5$. As suggested by B\&T, these differences should be attributed to different boundary conditions at the
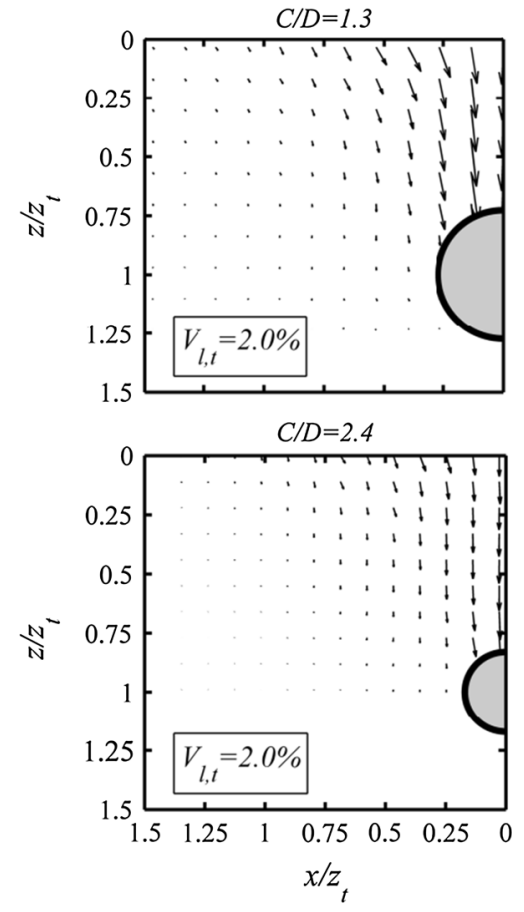

Fig. 2 Displacement fields measured during centrifuge tests performed by Marshall et al. (2012)

tunnel. The B\&T experiment included a rigidly lined model tunnel that imposes a concentric deformation pattern to the tunnel periphery (resulting in a displacement control boundary condition); the Marshall et al. model tunnel consisted of a fluid-filled flexible latex membrane, with no strict imposition of the lining deformation pattern during tunnel volume loss (achieved by water extraction). In the latter case, the equilibrium condition between the soil and the fluidfilled membrane controls the resulting shape of the tunnel lining at every stage of tunnel volume loss. Given that the soil used in the two series of experiments was relatively similar, the data in Fig. 1 would suggest that the tunnel lining boundary condition in the B\&T experiments are responsible for the wider settlement troughs above the tunnel compared to those from Marshall et al., which were characterized by a localised narrow collapse at the tunnel crown, as illustrated in Fig. 2. The data in Fig. 2 indicate that very little ground movement occurs at the sides of the tunnel if a concentric displacement pattern is not imposed on the tunnel lining. The impact of imposed displacements in these regions in the B\&T tests must relate to the observation of wider settlement troughs, 


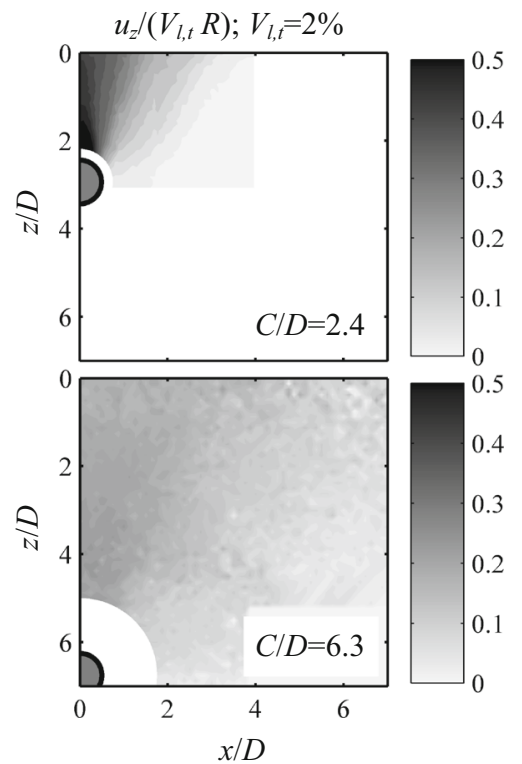

Fig. 3 Normalised vertical displacements induced by tunnel volume loss; fluid-filled tunnel with flexible rubber lining

especially at depths nearer the tunnel. Moreover, Fig. 1 illustrates that the profiles of $K$ with depth measured by B\&T are very similar at both $C / D$ ratios. The profiles estimated according to Marshall et al. (2012) are noted to change in both shape and magnitude. Therefore, using a concentrically

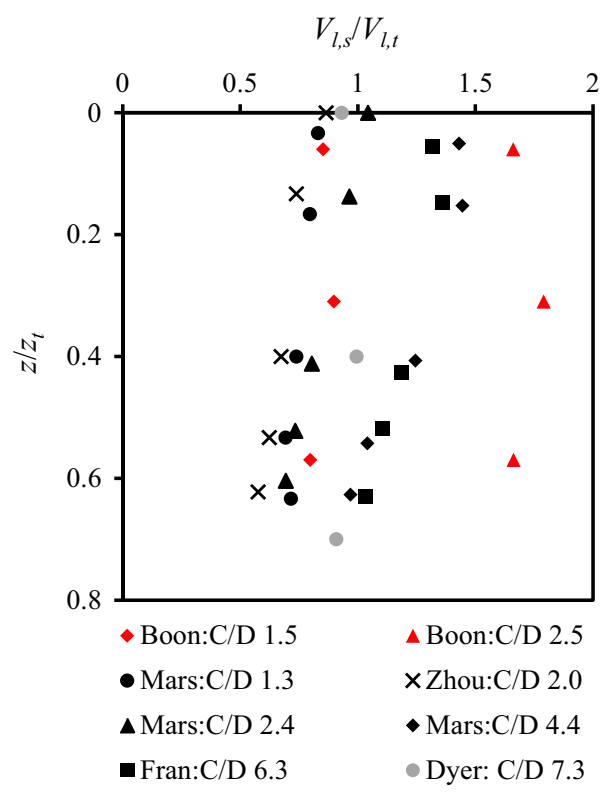

Fig. 4 Ratio between soil and tunnel volume loss with normalized depth at different values of $C / D$ contracting tunnel appears to result in $K$ distributions that are less affected by the $C / D$ ratio than when the tunnel boundary condition causes volume loss deformations concentrated at the tunnel crown.

There are also some important similarities between the deformation patterns predicted by both tunnel modelling techniques. The two datasets suggest a similar consequence of the $C / D$ ratio. Figure 3 shows the normalised vertical settlements $\left(u_{z} /\left(V_{l, t} R\right)\right.$, which allows comparison of displacements between tests with different sized tunnels) for $C / D=2.4$ (Marshall et al. 2012) and $C / D=6.3$ (Franza et al. 2016); the latter research used the same type of model tunnel as Marshall et al. 2012. Figure 3 illustrates that the chimney-like mechanism suggested for coarse soils by Cording (1991) describes the deformation pattern induced by shallow tunnels $(C / D<4)$, whereas for tunnels with $C / D>4$, the ground movements spread outwards from the tunnel without a zone of major settlement at the tunnel centreline. B\&T suggest that the chimney-like mechanism is also more noticeable for $C / D=1.5$ than for $C / D=2.5$.

Finally, the ratio between the soil volume loss, $V_{l, s}$, and the tunnel volume loss, $V_{l, t}=\Delta V / V_{O}$, was computed for the settlement curves provided in Fig. 11 of $\mathrm{B} \& \mathrm{~T}$ and is compared against other available data in Fig. 4. This ratio would be equal to unity for tunnels in undrained, constant volume soils. In sands, the variation from unity of $V_{l, s} / V_{l, t}$ at a certain depth is due to the cumulative effect of the soil volumetric strains beneath that level. Thus, to fully understand the relationships in Fig. 4, it is necessary to account for the volumetric strain distribution within the soil, which is related to the magnitude of shear strain and confining stress. The ratio $V_{l, s} / V_{l, t}$ is compared in Fig. 4 with data obtained from Dyer et al. (1996), Marshall et al. (2012), Zhou et al. (2014), and Franza et al. (2016). Both the results collected by B\&T and Marshall et al. (2012) illustrate an increase of $V_{l, s} / V_{l, t}$ with $C / D$ for a given value of $V_{l, t}$. This phenomenon was explained qualitatively in Marshall et al. (2012); for a given tunnel diameter and tunnel volume loss, since the magnitude of shear strains in relatively shallow tunnels is greater than for deeper tunnels, the soil reaches a dilatant state at lower magnitudes of tunnel volume loss for relatively shallow tunnels compared to deeper tunnels. However, the value of $V_{l, s} / V_{l, t}$ for the B\&T test with $C / D=2.5$ is quite high when compared with data for deeper tunnels $(\mathrm{Cl}$ 
$D>4)$. This is probably due to the fact that the concentric displacement control tunnel modelling technique used by B\&T induces low-level shear strains in the soil around the tunnel, which cause contractive soil behaviour within an extended area around the tunnel. This is supported by the data in Fig. 1, where the B\&T subsurface settlement curves are wider than those of Marshall et al. (2012), confirming that a greater volume of soil undergoes shearing around the tunnel for the concentric displacement control tunnelling technique. The larger zone of soil affected by tunnelling in the B\&T tests would undergo lower levels of shearing than the more localised zone in the Marshall et al. tests. This results in larger values of $V_{l, s} / V_{l, t}$ for B\&T both at the surface and subsurface. Note that the ratio $V_{l, s} / V_{l, t}$ computed from B\&T data is fairly constant with depth, indicating that most of the contractive behaviour of the soil is localised at or below the level of the tunnel. On the other hand, the water-filled flexible lining model tunnel results in negligible strains at the tunnel invert and springline (illustrated in Fig. 2), and the displacement mechanism is characterized by bands of high shear strains starting from the shoulders of the tunnel and developing towards the surface (Marshall et al. 2012). Similar strain distributions characterised by bands of high shear strains at the tunnel shoulder were suggested by Cording and Hansmire (1975) and Schuller and Schweiger (2002). This explains the decrease of $V_{l, s} / V_{l, t}$ with $z / z_{t}$ for the data sets in Fig. 4.

In general, it is not clear which boundary condition is more appropriate for the simulation of tunnelling in sands. However, previous researchers have suggested an oval-shaped (i.e. eccentric) tunnel volume loss distribution in clays resulting in small displacements at the tunnel springline and negligible displacements at the invert (Loganathan and Poulos 1998). This ovalshaped mechanism was successfully implemented by Cheng et al. (2007) in a numerical model of tunnelling-induced movements based on a displacement controlled approach (i.e. displacement boundary conditions were imposed at the tunnel periphery). This displacement controlled approach has been applied in several papers regarding tunnel-structure interaction. Furthermore, the coefficient of lateral earth pressure, $K$, is generally lower in sands than in clays, which suggests that horizontal movements at the tunnel springline should be lower in sands than in clays. Therefore, inducing a uniform radial tunnel contraction (with equal contraction at the tunnel crown, springline and invert) may not be realistic. It would be interesting to evaluate the trend of settlement trough shape (i.e. $K$ ) and $V_{l, s} / V_{l, t}$ with the methodology adopted by $\mathrm{B} \& \mathrm{~T}$ for $C / D>2.5$ to further study the effects of differing tunnel modelling techniques.

Open Access This article is distributed under the terms of the Creative Commons Attribution 4.0 International License (http:// creativecommons.org/licenses/by/4.0/), which permits unrestricted use, distribution, and reproduction in any medium, provided you give appropriate credit to the original author(s) and the source, provide a link to the Creative Commons license, and indicate if changes were made.

\section{References}

Boonsiri I, Takemura J (2015) Observation of ground movement with existing pile groups due to tunneling in sand using centrifuge modelling. Geotech Geol Eng 33(3):621-640

Cheng CY, Dasari GR, Chow YK, Leung CF (2007) Finite element analysis of tunnel-soil-pile interaction using displacement controlled model. Tunn Undergr Space Technol 22(4):450-466

Cording EJ (1991) Control of ground movements around tunnels in soil. In: Proceeding of 9th Pan-American conference on soil mechanics and foundation engineering, Valparaiso, vol 4. pp 2195-2244

Cording EJ, Hansmire WH (1975) Displacement around soft ground tunnels. In: Proceeding of 5th Pan-American conference on soil mechanics and foundation engineering, Buenos Aires. pp 571-632

Dyer MR, Hutchinson MT, Evans N (1996) Sudden valley sewer: a case history. In: Proceedings of international symposium on geotechnical aspects of underground construction in soft ground, London. pp 671-676

Franza A, Marshall AM (2015) Analytical investigation of soil deformation patterns above tunnels in sandy soil. In: Proceedings of XVI ECSMGE geotechnical engineering for infrastructure and development, Edinburgh. pp 467-472

Franza A, Zhou B, Marshall AM (2016). The effects of relative tunnel depth and volume loss on vertical settlements above tunnels in dense sands. In: Proceedings of the fourth GeoChina international conference, 260 GSP, Shandong. pp 125-132

Loganathan N, Poulos HG (1998) Analytical prediction for tunneling-induced ground movements in clays. J Geotech Geoenviron Eng 124(9):846-856

Marshall AM, Farrell RP, Klar A, Mair RJ (2012) Tunnels in sands - the effect of size, depth, and volume loss on greenfield displacements. Geotechnique 62(5):385-399

Moh ZC, Hwang RN, Ju DH (1996) Ground movements around tunnels in soft ground. In: Proceeding of international symposium geotechnical aspects of underground construction in soft ground, London. pp 725-730

Schuller H, Schweiger HF (2002) Application of a Multilaminate Model to simulation of shear band formation in NATM-tunnelling. Comput Geotech 29(7):501-524 
Sugiyama T, Hagiwara T, Nomoto T, Nomoto M, Ano Y, Mair RJ, Bolton MD, Soga K (1999) Observations of ground movements during tunnel construction by slurry shield method at the docklands light railway Lewisham extension-East London. Soils Found 39(3):99-112
Zhou B, Marshall AM, Yu HS (2014). Effect of relative density on settlements above tunnels in sands. In: Proceeding of 2014 GeoShanghai international congress: tunneling and underground construction, 242 GSP, Shanghai. pp 96-105 